\title{
Eye movement analyses indicate the underlying reading strategy in the recovery of lexical readers
}

\author{
Irene Ablinger ${ }^{1,2}$, Walter Huber ${ }^{2}$, and Ralph Radach ${ }^{3}$ \\ ${ }^{1}$ Section Neuropsychology, Department of Neurology, RWTH Aachen \\ University Hospital, Aachen, Germany \\ ${ }^{2}$ Section Clinical Cognition Research, Department of Neurology, RWTH \\ Aachen University Hospital, Aachen, Germany \\ ${ }^{3}$ Department of General and Biological Psychology, University of Wuppertal, \\ Wuppertal, Germany
}

\begin{abstract}
Background: Psycholinguistic error analysis of dyslexic responses in various reading tasks provides the primary basis for clinically discriminating subtypes of pathological reading. Within this framework, phonology-related errors are indicative of a sequential word processing strategy, whereas lexical and semantic errors are associated with a lexical reading strategy. Despite the large number of published intervention studies, relatively little is known about changes in error distributions during recovery in dyslexic patients.
\end{abstract}

Aims: The main purpose of the present work was to extend the scope of research on the time course of recovery in readers with acquired dyslexia, using eye tracking methodology to examine word processing in real time. The guiding hypothesis was that in lexical readers a reduction of lexical errors and an emerging predominant production of phonological errors should be associated with a change to a more segmental momentto-moment reading behaviour.

Methods \& Procedures: Five patients participated in an eye movement supported reading intervention, where both lexical and segmental reading was facilitated. Reading performance was assessed before (T1) and after (T2) therapy intervention via recording of eye movements. Analyses included a novel way to examine the spatiotemporal dynamics of processing using distributions of fixation positions as different time intervals. These subdistributions reveal the gradual shifting of fixation positions during word processing, providing an adequate metric for objective classification of online reading strategies.

Outcome \& Results: Therapy intervention led to improved reading accuracy in all subjects. In three of five participants, analyses revealed a restructuring in the underlying reading mechanisms from predominantly lexical to more segmental word processing. In contrast, two subjects maintained their lexical reading procedures. Importantly, the fundamental assumption that a high number of phonologically based reading errors must be associated with segmental word processing routines, while the production of lexical errors is indicative of a holistic reading strategy could not be verified.

Conclusions: Our results indicate that despite general improvements in reading performance, only some patients reorganised their word identification process. Contradictive

Address correspondence to: Irene Ablinger, Section Neuropsychology/Clinical Cognition Research, Department of Neurology, RWTH Aachen University Hospital, Aachen, Germany. E-mail: iablinger@, ukaachen.de

The present investigation was supported by the German Science Foundation, DFG (HU 292/9-2). Two anonymous reviewers provided very helpful feedback on an earlier version of this paper. We thank all patients for participating in our study. 
data raise doubts on the validity of psycholinguistic error analysis as an exclusive indicator of changes in reading strategy. We suggest this traditional approach to combine with innovative eye tracking methodology in the interest of more comprehensive diagnostic strategies.

Keywords: Acquired dyslexia; Aphasia; Eye movement; Reading strategy; Reading treatment.

Healthy reading is characterised by automatised and overlearned interactions of lexical and sublexical reading processes (Cherney, 2004; Coltheart, 2006, 2012; Coltheart, Rastle, Perry, Langdon, \& Ziegler, 2001; Houghton \& Zorzi, 2003; Rapcsak, Henry, Teague, Carnahan, \& Beeson, 2007) which enable skilled readers to identify familiar as well as unfamiliar words. Patients with acquired central dyslexia do not show this well-coordinated interaction of both mechanisms; instead, they pathologically rely predominantly on one of the two reading routes.

Traditionally, psycholinguistic error analysis of dyslexic responses in various reading tasks provides the basis for clinically discriminating subtypes of reading impairment (Coltheart, 1980; Greenwald, 2000; Morton \& Patterson, 1980; Patterson, Marshall, \& Coltheart, 1985). According to this approach, deep dyslexic patients show a complete failure of pseudoword reading due to severely impaired sublexical reading procedures. Word identification relies on a secondary, often error prone, lexical-semantic reading route. Hence, typical features of deep dyslexia are semantically and visually guided lexical errors, morphological and phonological errors, and the appearance of a concreteness effect (i.e., concrete words are read significantly better than abstract words; e.g., Buchanan, McEwen, Westbury, \& Libben, 2003; Coltheart, 1980; Rastle, Tyler, \& Marslen-Wilson, 2006). Phonological dyslexia is considered to be an alleviated form of deep dyslexia. Both disorders are widely assumed to represent two end points on a single severity-based dyslexia continuum and they exhibit a high overlap of dyslexic symptoms (Crisp, Howard, \& Lambon Ralph, 2011; Crisp \& Lambon Ralph, 2006; Friedman, 1996). The prevalence of the relevant erroneous responses is dependent on the interaction and severity of the phonological and semantic impairment (Crisp et al., 2011; Crisp \& Lambon Ralph, 2006).

In contrast, patients with surface dyslexia rely on a relatively intact sublexical reading route. Difficulties are manifested in direct access of orthographic representations with exceptional challenges when reading words with irregular orthography. Reading is characterised by apparent attempts to sequentially process words from left to right. This impairment is generally assumed to mainly result in phonological reading errors, although visually guided lexical errors can also be observed (e.g., Patterson et al., 1985).

Over the last two decades, numerous single case studies have described rehabilitation approaches of patients with acquired dyslexia. Treatment of acquired reading disorders is generally focused on individual deficits and residual capabilities, attempting to strengthen lexical, semantic, and/or phonological representations (Ablinger et al., submitted; Bowes \& Martin, 2007; Cherney, 2004; Coltheart \& Byng, 1989; De Partz, 1986; Friedman \& Nitzberg Lott, 2002; Friedman \& Robinson, 1991; Kiran, Thompson, \& Hashimoto, 2001; Leff \& Behrmann, 2008; Mitchum \& Berndt, 1991; 
Nickels, 1992; Stadie \& Rilling, 2006; Weekes \& Coltheart, 1996; Yampolsky \& Waters, 2002).

Irrespective of the particular method of treatment, therapy outcome is usually assessed via improved reading accuracy (e.g., Friedman \& Nitzberg Lott, 2002; Friedman \& Robinson, 1991; Yampolsky \& Waters, 2002) and changes in reading behaviour. In this context, only a minority of studies have reported the distribution of errors over various categories. However, this specification is useful, as changes in error distribution over the time course of recovery may reveal critical information on the underlying reading procedure (see Ablinger, Huber, Schattka, \& Radach, 2013; Ablinger et al., submitted; Schattka, Radach, \& Huber, 2010). Following this logic, segmental reading is characterised by a high number of segmental errors, whereas predominant lexical and semantic errors are indicative of lexical reading.

Reading error classification can also be used to infer changes in reading strategies. As an early example, De Partz (1986) reported modified reading behaviour in a previously deep dyslexic reader after a 9-month intensive therapy intervention. In spite of a still existing discrete imageability deficit, a regularity effect, characteristic for surface dyslexia, emerged in this patient. In line with this development, errors typical for deep dyslexia, such as semantic errors, nearly disappeared while errors resulting from a misapplication of grapheme-phoneme transcoding rules became more frequent. A change from lexical to segmental reading was also described by Nolan and coworkers (Nolan, Volpe, \& Burton, 1997). Their patient suffered from a traumatic head injury und initially showed clear symptoms of deep dyslexic reading with poor grapheme-phoneme conversion abilities. However, 4 months after his injury, he presented mild surface dyslexia with a substantial regularity effect as well as phonologically and visually based reading errors.

During the past decade, the recording and analysis of eye movements (see Radach \& Kennedy, 2004, 2013; Rayner, 2009, for comprehensive overviews) has proved extremely useful to gain a better understanding of visuomotor and linguistic mechanisms in pathological reading with peripheral and central disorders (e.g., Ablinger et al., 2013; Behrmann, Shomstein, Black, \& Barton, 2001; Johnson \& Rayner, 2007; Rayner \& Johnson, 2005; Schattka et al., 2010).

This research is challenging due to the complexities of eye movement behaviour in pathological reading, with patients typically engaging in multiple fixations and reading passes. Our team has attempted to counter these problems in two related ways. First, we have developed a reading task in which we arrange unrelated target words in a line by line format, removing the higher order processing demands of sentence reading, but preserving the dynamic nature of word by word processing (Schattka et al., 2010). Second, our principal way of analysing data from patients with severe reading difficulties relies on a combination of spatial and temporal eye movement parameters, allowing us to quantify the use of different reading strategies (Ablinger et al., 2013, submitted). More specifically, the multiple fixations made during an attempt to read a target word are divided into equal bins (usually terciles are used, corresponding to the 1st, 2nd, and 3rd thirds of all fixations which are made) and the resulting partial distributions of fixation positions computed (see below, for a more detailed description). This analysis strategy is guided by the idea that segmental reading is characterised by gradually moving fixations through the word from left to the right, while in lexical reading fixation positions tend to accumulate close to word centre 
(Ablinger et al., 2013; Schattka et al., 2010). ${ }^{1}$ Building on this work, we recently developed an eye-movement-based treatment method for patients with central dyslexia. The method uses saccade contingent display changes to trigger either sequential or holistic word processing (see below for details), with the aim of strengthening both the more impaired and the better preserved processing route, as well as improving flexibility in utilising both reading routes in patients with central dyslexia (Ablinger et al., submitted). All subjects of that study benefited from therapy intervention; total reading time, the needed number of fixations to identify the target, and reading accuracy improved significantly. While seven of eight participants maintained or even optimised the underlying reading procedure, only one patient changed his underlying reading strategy as a consequence of therapy intervention. He switched from a clearly lexical to an explicit sequential reading procedure. Interestingly, he is also the only lexical reader who showed a considerable reduction of lexical errors after reading intervention.

In the present study, we substantially extend this work to examine in more detail the dynamics of reading recovery in a sample of patients all diagnosed as lexical readers. Both conventional linguistic error analyses and eye tracking methodology were utilised to study word reading strategies at two time points, before and after therapy intervention. The focus of interest was the question whether linguistic error type and changes in distributions over various error types are associated with changes in real-time reading behaviour as indicated by eye movements. Our theoretical starting point was the hypothesis that in lexical readers a reduction of lexical errors and an emerging predominant production of phonological errors should be associated with a change to a more segmental moment-to-moment reading behaviour. This work provides an extension of the well-established approach to characterise procedures of oral reading based on overt articulation. In addition, we include online eye tracking methodology to obtain information about processes of word identification in real time (Radach \& Kennedy, 2013; Rayner, 2009).

\section{METHODS}

\section{Participants}

Five subjects with manifested acquired dyslexia, all in the chronic stage of aphasia, participated in this study. Patients were recruited from the Aachen aphasia ward, where they received an intensive 7-week treatment (Huber, Springer, \& Willmes, 1993). All participants were native German speakers, had normal or corrected to normal vision and were premorbidly right-handed (Edinburgh Inventory, Oldfield, 1971). Type and severity of aphasia were determined based on the Aachen Aphasia Test

\footnotetext{
${ }^{1}$ The validity of fixation positions within words as indicators of lexical and sub-lexical processing has been confirmed in research conducted from a number of different perspectives. On a fundamental level, it appears clear that the centre of words provides the functional target for incoming initial saccades into words (Engbert \& Krügel, 2010; Rayner, 1979). Looking at eye movements within words, research on the reading of complex words has indicated that saccade landing positions are sensitive to fine grain processing strategies on the orthographic and morphological level (e.g., Hyönä, Bertram, \& Pollatsek, 2004; Inhoff, Radach, \& Heller, 2000). Systematic relations between fixation positions and fixation durations have been documented (Radach \& Heller, 2000; Vitu, McConkie, Kerr, \& O'Regan, 2001), and the more sequential nature of word processing in developing readers has been shown to clearly correspond to more within-word refixations and larger oculomotor word length effects (Huestegge, Radach, Corbic, \& Huestegge, 2009; Joseph, Liversedge, Blythe, White, \& Rayner, 2009).
} 
(AAT) performance profiles (Huber, Poeck, Weniger, \& Willmes, 1983; Willmes, 1985) before and after therapy. The etiology was vascular in four patients, one suffered from a craniocerebral injury (CCI). A detailed description is provided in Table 1.

\section{Procedure and material}

All five participants were included in an eye-movement-based reading intervention (for methodological details, see Ablinger et al., submitted; key elements are reported below). In the present study, we sought to determine whether a specific therapy approach facilitates a change in the underlying reading strategy. For this reason, we determined the preferred reading strategy for every participant on the basis of oculomotor behaviour before and after reading intervention (T1, T2). Participants were asked to read aloud a set of 150 target words while eye movements and articulation were recorded simultaneously. Target words were individually selected during baseline examination (T1), where a total of 400 potential targets had to be read twice during two consecutive sessions. Words that could not be named or showed an extremely inflated reading time were included into the individualised item pool. All of these items were nouns varying in word length from six to nine letters.

\section{Classification of reading strategy}

To classify the underlying reading strategy, we utilised a data analysis technique that has proved very useful in our recent work (Ablinger et al., 2013, submitted). More specifically, we divided the total number of fixations per target word into three equal bins (terciles) and computed the resulting distributions of fixation positions. Comparing these subdistributions reveals the gradual shifting of fixation positions over the time course of word processing, providing an adequate metric for objective classification of reading strategies. A patient using a sequential reading strategy gradually moves fixations through the word from left to right, resulting in three spatially distant peaks in position distributions spread out between word beginning and word end. In contrast, lexical readers typically produce subdistributions with fixation positions accumulating near the word centre.

\section{Therapy procedure}

Our approach to therapy follows the principle of combined treatment by stimulating both lexical and segmental word processing routes. The lexical reading method was designed to stimulate whole word processing. To this end, eye movement contingent display changes were employed to force fixation of the word centre (see Inhoff, Eiter, \& Radach, 2005; Schotter, Angele, \& Rayner, 2012, for discussions of saccade contingent display methods). Critically, as soon as a fixation was detected outside of the centre region, all letters outside of this region became masked. After fixating within the central field for $3,000 \mathrm{~ms}$, the patient was confronted with a corrective feedback about her/his reading performance. In the segmental therapy approach, serial processing of target items was supported by a gradual presentation of word segments. Depending on word length, words were displayed in two or three segments. The complete unmasked word remained on the screen for 3,000 ms. After completion of each item, the patient was confronted with corrective feedback about her/his reading performance. 


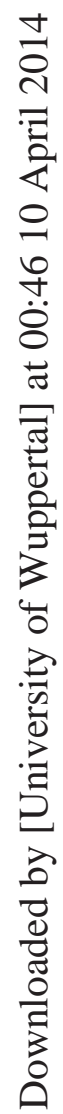






\section{Apparatus and procedure}

To examine reading performance, target words were presented on the computer screen arranged in lines similar to normal sentence reading. This alignment preserves the normal spatial configuration found in text reading so that visual processing demands are close to a natural reading situation. Importantly, the task of reading a sequence of well-controlled nouns avoids higher order post-lexical processing on the sentence and text level that often overwhelms patients with severe reading disabilities. This methodology was used successfully in our laboratory to examine patients suffering from mild to moderate central dyslexia in the context of aphasia and peripheral dyslexia, again showing sensitivity to visual and cognitive processing demands (Ablinger et al., 2013, submitted; Schattka et al., 2010).

Eye movements were recorded using an SR EyeLink 1,000 video-based eye tracking system. The registration is based on infrared light reflection from pupil and cornea at a sampling rate of $1,000 \mathrm{~Hz}$. Viewing was binocular but eye movements were recorded from the right eye only. Head position was stabilised with a forehead rest and small movements were compensated online. The system calibrates eye position using an array of three black dots placed equidistantly on a horizontal target line. These targets are presented individually in fixed order and subjects are asked to fixate each dot, which is automatically controlled by the eye tracking system. Successful calibration was immediately followed by a validation routine that determined the stability and accuracy of the initial measurement.

The target words were displayed in black on a grey background using a 21-inch monitor running at a resolution of $1,680 \times 1,050$. Text was presented in nonproportional 15 point Courier font corresponding to 12 pixels per letter horizontally. At a viewing distance of $54 \mathrm{~cm}$, each character subtended approximately $0.5^{\circ}$ of visual angle. Blanks before words had the width of a character. After the first calibration, the reading task was demonstrated using one line of eight practice items. Participants were asked to fixate a cross at the left end of the line before the eight written words were shown simultaneously. This served as a signal to start reading aloud all eight words in a sequence. When finished, the participant fixated a string of $\mathrm{x}(\mathrm{xxXxx})$ at the end of the line and pressed a key on a gamepad which caused the stimuli to disappear. During each session, verbal expressions were digitally recorded on a PC (Adobe Audition, 1.5) for subsequent linguistic analysis.

\section{Data selection and analysis}

A target word was included in data analysis when a fixation fell on at least one of its constituent letters or on the blank space preceding it. Data were organised in terms of pairs of incoming saccades and following fixation durations. Observations containing blinks or fixation durations shorter than $70 \mathrm{~ms}$ or longer than 2,000 ms, and those outside of three standard deviations of the mean were excluded from analyses. In addition to these standard procedures (Inhoff \& Radach, 1998), we also discarded observations with just one fixation per gaze to eliminate cases where eyes have briefly slipped over word boundaries. The first and last word on the line served as fillers and were not considered in any data analyses. In the current study, we used total reading time (the summed duration of all fixations within the target word including later rereading), and total number of fixations to characterise the reading process of our 
dyslexic subjects (see Ablinger et al., 2013; Johnson \& Rayner, 2007; Rayner \& Johnson, 2005, for similar data analysis procedures).

Eye movement data were analysed with the software Eye Movement Data Analyser (EyeMap; Tang, Reilly, \& Vorstius, 2012) and subsequent statistical analyses were computed using IBM SPSS Statistics 21 software. Performance at different testing points was assessed with the nonparametric Wilcoxon signed-rank test. Reading accuracy between testing points was examined with the McNemar's test. All statistical analyses are based on one-sided testing.

In line with prior research, we also conducted a standard linguistic error analysis. Reading errors were classified into four categories: segmental phonological errors (including neologistic responses with high as well as low phonological similarity to the target), lexical errors (including lexical responses with and without visual (orthographic) similarity to the target), semantic errors (including responses with semantic relation to the target item), and omissions (including no responses, abortions, and perseverations).

\section{RESULTS}

In the following section, we first report standard error analyses describing overt reading behaviour in all five subjects. We then refer to eye movement patterns in terms of word viewing time and number of fixations to quantify the effectiveness of word reading. Finally, we examine the spatiotemporal dynamics of word reading to determine reading strategies used by our patients over the course of the recovery progress.

\section{Overt reading behaviour}

In all subjects, oral word and pseudoword reading was poor before therapy intervention. While word reading improved significantly in all participants, there were no changes in pseudoword reading patterns in any of the patients. Table 2 provides a summary of oral reading responses.

TABLE 2

Accuracy and error distribution in word reading. Accuracy for pseudowords

\begin{tabular}{|c|c|c|c|c|c|c|c|}
\hline & \multicolumn{6}{|c|}{ Words $(n=150)$} & \multirow{2}{*}{$\begin{array}{l}\text { Pseudowords } \\
\qquad \begin{array}{c}(n=80) \\
\text { Accuracy } \\
(\%)\end{array}\end{array}$} \\
\hline & Test & $\begin{array}{c}\text { Accuracy } \\
(\%)\end{array}$ & $\begin{array}{c}\text { Segmental } \\
\text { phonological errors } \\
(\%)\end{array}$ & $\begin{array}{c}\text { Lexical } \\
\text { errors }(\%)\end{array}$ & $\begin{array}{l}\text { Semantic } \\
\text { errors }(\%)\end{array}$ & $\begin{array}{c}\text { Omissions } \\
(\%)\end{array}$ & \\
\hline \multirow[t]{2}{*}{ DH } & $\mathbf{T 1}$ & $16(10.7)$ & $41(27.3)$ & $68(45.3)$ & $11(7.3)$ & $14(9.3)$ & $1(1.3)$ \\
\hline & $\mathbf{T} 2$ & $66(44.0)^{*}$ & $28(18.7)^{*}$ & $30(20.0)^{*}$ & $1(0.6)^{*}$ & $17(11.3)$ & 0 \\
\hline \multirow[t]{2}{*}{ SHJ } & T1 & $0(0)$ & $6(4)$ & $43(28.6)$ & $9(6.0)$ & $92(61.3)$ & 0 \\
\hline & $\mathbf{T} 2$ & $72(48.0)^{*}$ & $10(7)$ & $45(30.0)$ & $5(3.3)$ & $18(12.0)^{*}$ & 0 \\
\hline \multirow[t]{2}{*}{ ST } & $\mathbf{T 1}$ & $0(0)$ & $22(14.7)$ & $13(8.7)$ & $0(0)$ & $115(76.7)$ & 0 \\
\hline & $\mathbf{T} 2$ & $22(14.7)^{*}$ & $26(17.3)$ & $5(3.3)^{*}$ & $0(0)$ & $97(64.7)^{*}$ & 0 \\
\hline \multirow[t]{2}{*}{ KM } & $\mathbf{T 1}$ & $0(0)$ & $19(12.7)$ & $51(34.0)$ & $10(6.7)$ & $70(46.7)$ & $0(0)$ \\
\hline & $\mathbf{T} 2$ & $52(34.7)^{*}$ & $46(30.7)^{*}$ & $44(29.3)$ & $6(4.0)$ & $2(1.3)^{*}$ & $3(3.8)$ \\
\hline \multirow[t]{2}{*}{ SW } & $\mathbf{T 1}$ & $18(12.0)$ & $56(37.3)$ & $13(8.7)$ & $4(2.7)$ & $53(35.3)$ & $1(1.3)$ \\
\hline & $\mathbf{T} 2$ & $89(59.3)^{*}$ & $35(23.3)^{*}$ & $8(5.3)^{*}$ & $2(1.3)$ & $15(10.0)^{*}$ & 0 \\
\hline
\end{tabular}

*Significant change in error distribution from T1 to T2, $p<.05$, McNemar's test. 
DH's reading was relatively fluent with some syllable-by-syllable utterances. At $\mathrm{T} 1$, he already showed some correct responses. His reading was dominated by lexically based responses and to a lesser part by phonologically guided reading errors. Most of his semantic errors showed a visual (orthographic) similarity to the target. At T2, lexical errors decreased significantly, so that the proportion of lexically and segmental phonologically based errors became relatively balanced.

SHJ produced a high proportion of omissions at T1. The majority of his verbal utterances were lexical errors with visual similarity to the target. He showed some semantic errors, some of which appeared visually (orthographically) related to the target. All of his utterances were quite fluent. After reading intervention at T2, his oral utterances suggested a change in his reading behaviour. Now only a few correct responses were articulated immediately. In most cases, he needed several attempts to identify the first graphemes (sometimes they were spelled alphabetically). This eventually resulted either in correct responses or lexical errors with visual similarity to the target.

At T1, most of ST's responses were omissions; in addition, he also produced segmental phonological errors and lexical errors. Overall, ST appeared quite fast in scanning the word line. After therapy intervention at T2, omissions remained the main error type, but reading accuracy had increased. Segmental phonological errors remained the main erroneous responses. At both testing points, responses were sometimes produced syllable by syllable. Due to mild impairments in terms of dysarthria and speech apraxia, some responses were imprecisely articulated.

KM's responses were only in part produced fluently. She often made several attempts to identify the first phoneme of a target word. These attempts predominantly resulted in lexical errors and omissions (including abortions and perseverations). At T2, the number of correct responses increased significantly. Segmental phonological errors and lexical errors were the main error type after therapy intervention.

SW showed some correct target word articulations at T1. However, most of his responses were omissions and segmental phonological errors. Independent of error type, he often needed several attempts to identify the target, and, similar to patient ST, his utterances were often produced in a syllable-by-syllable fashion. After reading intervention at $\mathrm{T} 2$, the number of correct responses increased significantly. In the majority of cases, responses were pronounced in and most frequently errors were still phonological in nature.

\section{Word viewing times before and after intervention}

Table 3 provides individual comparisons of total reading time and total number of fixation per word for T1 versus T2 along with adequate test statistics (nonparametric Wilcoxon signed-rank tests). The expected outcome would be a significant decrease in both parameters, indicating more effective word processing as result of therapybased improvement. However, this result was only observed in three out of five patients (DH, ST, and SW). In contrast, for two subjects (SHJ, KM), total reading time and total number of fixations increased significantly from T1 to T2. A possible explanation for this very interesting result can be derived from the distribution of reading errors reported in Table 3 . Since both patients produced a relatively high proportion of omissions before the beginning of reading intervention, the larger cognitive load invested at T2 may indicate that full word recognition was attempted 
TABLE 3

Number of fixations and total reading time (in ms) per word as a function of therapy intervention (standard deviations are given in parentheses)

\begin{tabular}{lcccccr}
\hline & $\begin{array}{c}\text { T1 Number of } \\
\text { fixations }\end{array}$ & $\begin{array}{c}\text { T2 Number of } \\
\text { fixations }\end{array}$ & $p$-Value & $\begin{array}{c}\text { T1 Total Reading } \\
\text { time in ms }\end{array}$ & $\begin{array}{c}\text { T2 Total reading } \\
\text { time in ms }\end{array}$ & $p$-Value \\
\hline DH & $14.6(7.0)$ & $11.8(6.0)$ & $<.001$ & $4,061(2,045)$ & $3,203(1,702)$ & $<.001$ \\
SHJ & $22.6(6.4)$ & $39.4(25.3)$ & $<.001$ & $6,809(2,049)$ & $12,428(8,112)$ & $<.001$ \\
ST & $11.1(5.1)$ & $6.3(2.7)$ & $<.001$ & $3,455(1,810)$ & $1,976(885)$ & $<.001$ \\
KM & $12.8(8.1)$ & $16.8(13.1)$ & $=.002$ & $3,253(2,173)$ & $4,606(3,323)$ & $<.001$ \\
SW & $31.7(22.7)$ & $21.6(16.0)$ & $<.001$ & $9,303(6,698)$ & $6,457(4,889)$ & $<.001$ \\
\hline
\end{tabular}

$p$-Values refer to nonparametric Wilcoxon signed-rank test.

in cases that at $\mathrm{T} 1$ would have resulted in premature cancellation of processing. Indeed, when only correct responses are included in the analyses, KM shows a comparable pattern as the other participants.

\section{Reading strategies over the course of therapy intervention}

The oculomotor behaviour during reading at both testing points served to determine individually preferred reading strategies. Analyses of fixation positions during the initial, middle, and final part of word processing indicated a predominantly lexical reading strategy in all patients at T1 (see Figure 1). In DH, KM, and SW, the spatial distance between the peaks of the first and the last third of the fixation distribution was two characters or less. Fixation positions accumulated to a central point, ideally, as observed with $\mathrm{DH}$ and $\mathrm{KM}$, close to or at the word centre. ST slightly deviated from an ideal pattern of lexical reading as his final third of fixation positions showed a second peak at letter position 5, indicating preferred lexical reading combined with some degree of sublexical processing. In SHJ, the asymmetric co-appearance of both reading routes is even more obvious. However, since the peak of the final subdistribution was located only one position right of the word centre the predominant reading strategy was determined as lexical.

Looking at T2, there is clear evidence that in three of five subjects (DH, ST, SHJ), a restructuring of the reading process occurred. In DH and ST, the peaks of all subdistributions were spatially spread out between word beginning and word end, indicating a transition towards much more sequential word processing. In SHJ, three clearly spatially marked fixation distributions emerged, located between word beginning and word centre. Apparently, word identification started with a sequential processing strategy but still included a substantial lexical component. In contrast, KM and SW maintained their lexical reading procedures at T2, as indicated by spatially overlapping subdistributions. Most fixations in their final third of word processing are clearly clustered left of the word centre. ${ }^{2}$

\footnotetext{
${ }^{2}$ Saccade landing sites were also plotted exclusively for trials with versus without correct responses, resulting in distributions very similar to those reported in Figure 1. This supplementary analysis suggested that there appears to be no direct relationship between real-time visuomotor behaviour and the success of word recognition as expressed in verbal responses.
} 

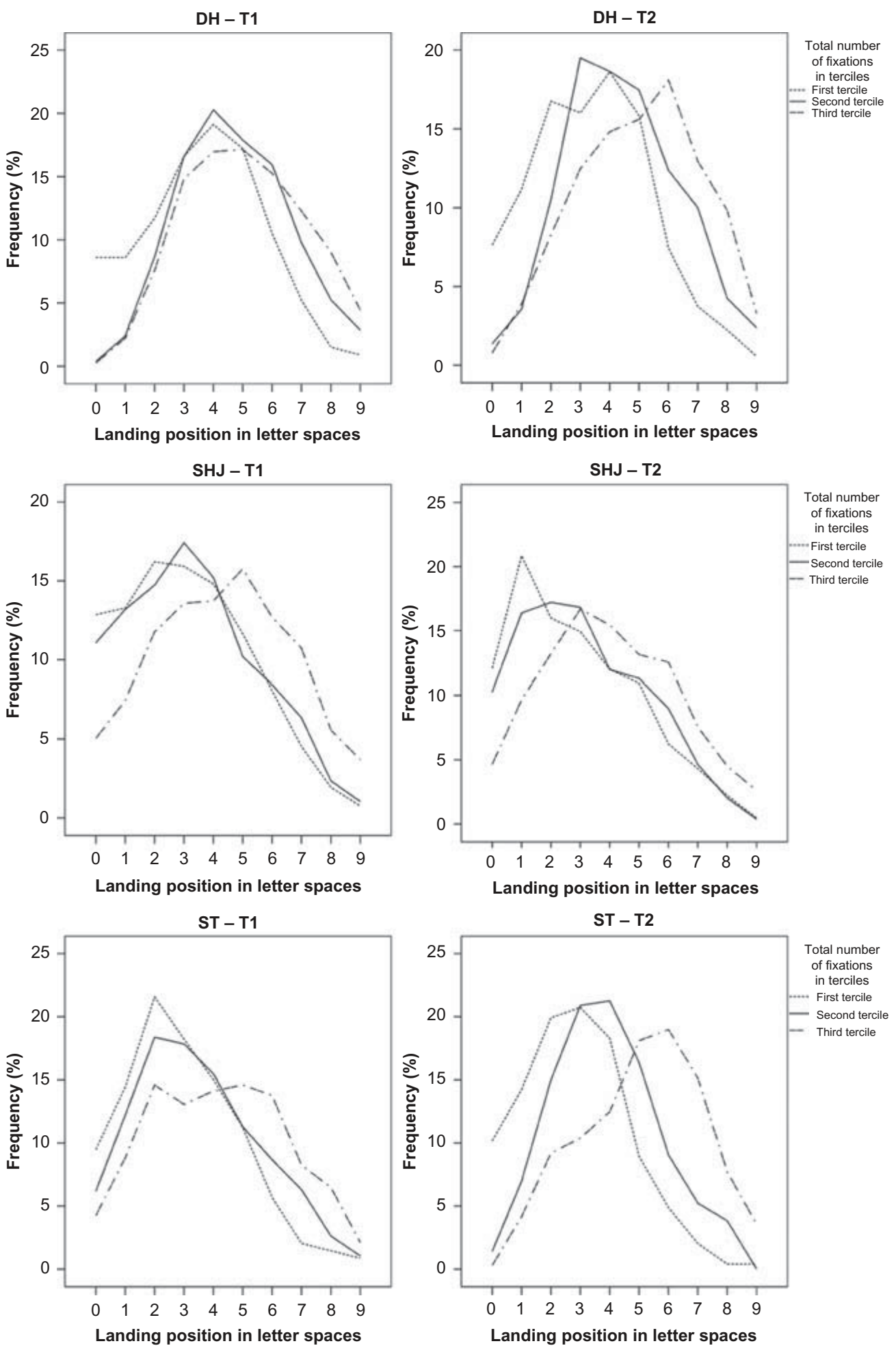

Figure 1. (a) Distribution of fixation positions at $\mathrm{T} 1$ and $\mathrm{T} 2$ partitioned into the first, second, and third part of word processing based on number of fixation terciles. Data for patients with a modified reading strategy, from initial lexical reading towards segmental reading. (b) Distribution of fixation positions at T1 and T2 partitioned into the first, second, and third part of word processing based on number of fixation terciles. Data for patients with a maintained lexical reading strategy.

For every participant, general language performance was reassessed by the AAT (Huber et al., 1983) at the end of their 7-week treatment in the Aachen aphasia ward. Performance in spontaneous speech, the Token Test, literary language, repetition, 

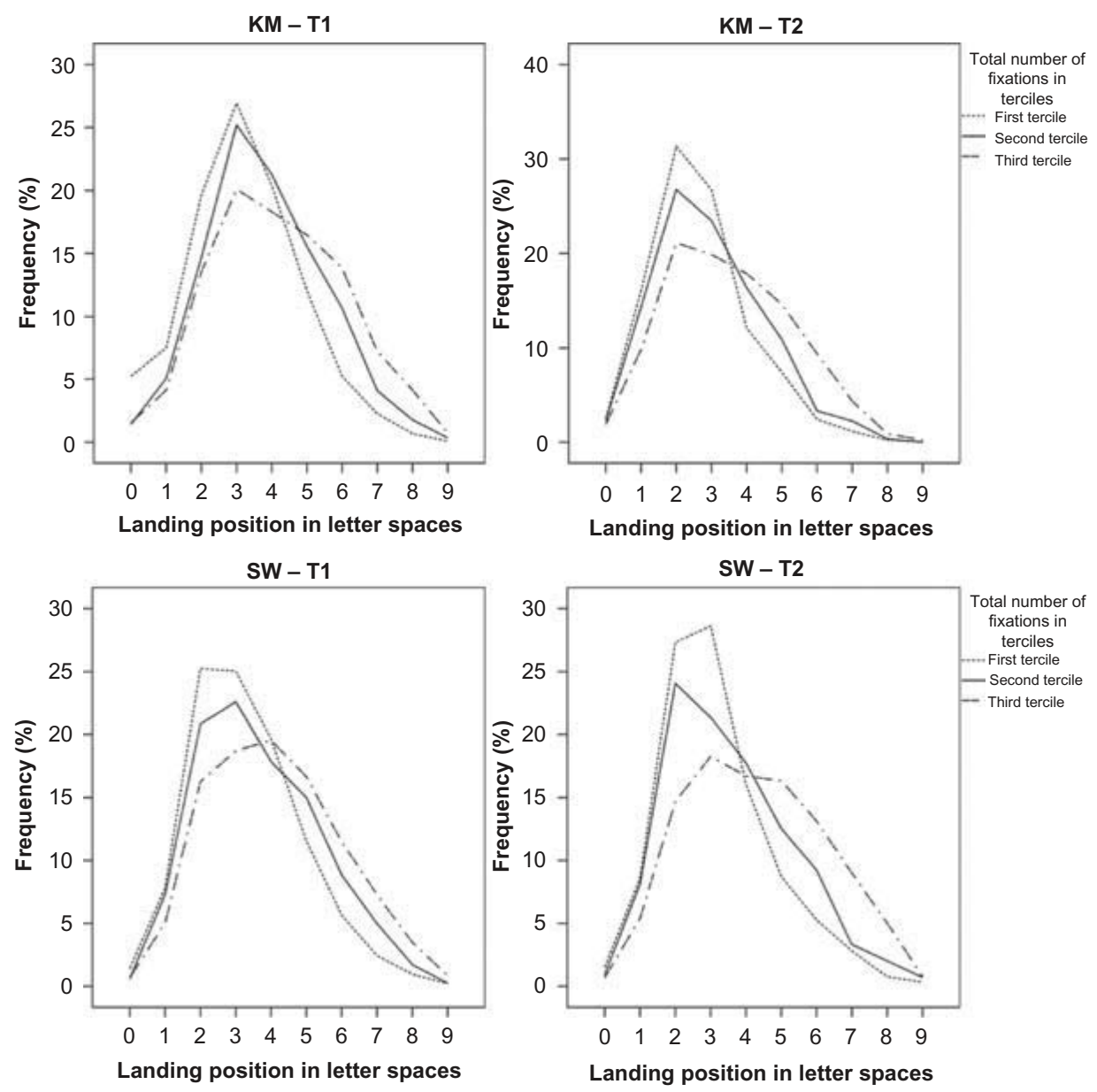

Figure 1. (Continued)

naming, and comprehension were assessed. Only two subjects (KM and SHJ) improved significantly in the subtest literary language. KM also showed significant improvements in naming, which was also part of an intensive treatment programme. In all other subjects all other subtests remained stable, or only improved marginally in comparison to the AAT pretest.

\section{DISCUSSION}

One major aim of the present study was to determine the relationship between changes in error distribution over the course of dyslexic recovery and reading strategies as indexed by visuomotor behaviour. Reading strategies were examined in five aphasic lexical readers before and after a specific therapy intervention. Innovative eye tracking methodology provided detailed information about minimal changes in real-time word identification processes.

On a general level, it is apparent that therapy intervention led to improved reading accuracy in all five subjects with chronic aphasia. Due to the chronic stage of disease, it appears exceedingly unlikely that spontaneous recovery contributed to the efficacy of our therapy. Indeed, assessment results indicated that none of the subjects improved performance in skills that were not part of our specific training, including repetition, comprehension, or naming (except for one patient who received a specific 
naming treatment). Only two subjects (KM and $\mathrm{SHJ}$ ) showed further improvements, but exclusively in literary language.

In conclusion, it appears safe to assume that therapy success can be attributed to our specific reading intervention. Word identification succeeded faster and was associated with a reduced number of fixations in three patients, DH, ST, and SW. In $\mathrm{KM}$ and $\mathrm{SHJ}$, baseline performance was characterised by the production of a relatively high proportion of omissions. As a consequence of treatment, the number of abandoned trials was substantially reduced, leading to a paradoxical increase in total reading time and number of fixations. When increased performance is observed, this may be attributed either to optimising predominant reading procedures or to the re-calibration of both reading routes so that the typical asymmetry between lexical and sequential reading is reduced. The first possibility appears to be typical for patients with pure alexia (e.g., Ablinger et al., 2013), whereas for central dyslexia, the situation is less clear.

Traditionally, a psycholinguistic error analysis of dyslexic responses in various reading tasks serves to classify the predominant reading strategy (Buchanan et al., 2003; Coltheart, 1980; Patterson et al., 1985; Rastle et al., 2006). Consequently, any modification in reading behaviour should be associated with changes in the observed error distribution. However, in most published studies, very little information is given about possible shifts of error distribution in relation to assumed reading strategies in the recovery process of dyslexic readers (see De Partz, 1986; Nolan et al., 1997, for notable exclusions).

The present work used a recently developed technique for objective classification of preferred reading strategies (Ablinger et al., 2013, submitted). Usually, in eye movement research on reading the analyses of temporal and spatial parameters is kept separate, based on the assumption that the decisions of when versus where to move are guided by different mechanisms (e.g., Liversedge \& Findlay, 2000). The standard way to report on the spatial aspect of eye movement control is to plot the distribution of the landing positions of incoming saccades over all letter positions within a target word (see Radach \& Kempe, 1993; Radach \& McConkie, 1998, for German data). The large number of fixations made by dyslexic patients during the processing of a single word makes it possible to partition the total fixation position distributions into subdistributions for different phases of word processing. This allows specifying where the focus of information acquisition is during each phase and, most importantly in the given context, it makes any shift in this focus of information intake transparent (see Radach, Inhoff, \& Heller, 2004, for a discussion of factors affecting saccade landing sites in normal reading).

Ideally, a patient using a sequential reading strategy should gradually move fixations through the word from left to right, resulting in spatially distant peaks in position distributions spread out between word beginning and word end. In contrast, an ideal lexical reader should produce subdistributions with fixation positions accumulating close to the word centre. The present study examined a sample of patients that all showed strong oculomotor evidence for a preferred lexical reading strategy before intervention. In addition to this dominant lexical processing mode, two patients (ST, SHJ) also demonstrated an additional sequential processing component.

In contrast, linguistic error analysis suggested only in three subjects a typical lexical reading pattern with predominant lexical errors at T1 (DH, SHJ, KM). Although ST also produced many lexical errors, the vast majority of erroneous verbal responses in both ST and SW were classified as segmental phonological errors. 
In line with traditional linguistic error analysis, this main error type would indeed indicate to a more sequential reading procedure.

Looking at changes in error distribution over the course of recovery, SW and SHJ remained more or less stable. Although lexical errors remained the main verbal response in SHJ, he was the only patient whose overt responses suggested a change in the underlying reading strategy. Omissions and lexical errors with visual similarity to the target were replaced by repeated attempts to identify the first graphemes. Although this very time consuming identification process succeeded in nearly $50 \%$ of the items, the modified reading behaviour often exceeded his capacities and led to the production of a relatively high number of visually based guessing errors (see also Friedman \& Nitzberg Lott, 2000). This overt behaviour corresponds nicely to the oculomotor data at T2. As fixation patterns suggest, sequential word identification was only present for the first half of the word. Additionally, the fact that SHJ rarely fixated to the right of the word centre is fully in line with the high number of guessing errors.

In KM, our specific reading intervention caused a significant reduction of omissions, while phonologically based reading errors increased. DH showed a considerable decrease in lexical errors at T2. For both patients, the proportion of lexical and segmental errors was quite counterbalanced. Nevertheless, fixation position analysis at $\mathrm{T} 2$ revealed a sustained lexical reading procedure after therapy intervention in $\mathrm{KM}$, and a reorganisation from lexical to segmental reading in $\mathrm{DH}$. We assume that the change in his oculomotor behaviour related to more successful sequential reading compared to the frequent lexical guessing errors observed at baseline.

A slight increase in segmental phonological errors and decrease in lexical errors at T2 were found in ST. Still, sequential phonological errors remained the main verbal erroneous responses at $\mathrm{T} 2$. Our intervention led to much more incremental reading as indicated by the spatial separation between peaks of fixation position subdistributions, clearly reflecting a change from lexical to segmental reading. This procedure presumably also structured and facilitated his verbal output, which was influenced by speech apraxia. In contrast, SW did not show any substantial changes in error distribution from $\mathrm{T} 1$ to $\mathrm{T} 2$, as segmental phonological errors remained the dominant error type. Similarly, fixation position analysis did not reveal a change in the underlying reading strategy.

Perhaps the most important result of the present work is that, with the exception of SHJ, data from all participants indicate discrepancies between word reading strategies suggested by linguistic error analysis and those documented with visuomotor data. This is especially clear in the case of SW, who maintained a strong tendency for targeting saccades at the word centre at both T1 and T2, even though his many segmental phonological errors would predict the opposite. On the other hand, ST clearly modified his online processing behaviour towards a more sequential strategy, a change that was not at all reflected in his overt error distribution. Our data indicate that readers who maintained a lexical strategy are slower and need more fixations compared to segmental readers for successfully identifying a word. The observed increase in word processing effort may at least partly be related to difficulties in access to lexical phonology.

To summarise, the hypothesis that a high number of phonologically based reading errors should be associated with online segmental word processing routines, while the production of lexical errors is indicative of a holistic reading (decoding) strategy, could not be verified in our visuomotor analyses. More 
generally, the data raise doubts on the common assumption that overt reading errors (and changes in error distribution) necessarily provide a reliable indicator for online word processing.

As a tentative conclusion, we suggest that when examining oral reading of patients with central dyslexia, features of the verbal output should be considered separate from real-time letter and word recognition routines (see, Inhoff, Solomon, Radach, \& Seymour, 2011; Vorstius, Radach, \& Lonigan, in press, for detailed discussions of visual and linguistic information processing in normal oral reading, for detailed discussions of visual and linguistic information processing in normal oral reading). The point is somewhat similar to the issue of verbal repetition, where it has been useful to differentiate between decoding and encoding routines in word processing (Ablinger, Abel, \& Huber, 2008; Baron, Hanley, Dell, \& Kay, 2008; Dell, Martin, \& Schwartz, 2007). It is our view that letter decoding and word recognition mechanisms are predominantly reflected in the observable behaviour of the eyes as letter and word processing progresses over time. On the other hand, psycholinguistic error analyses remain the gold standard for assessing the nature of encoding and production routines that determine the end result of word processing and the subsequent verbal responses. At both end of this spectrum processing may be of a more segmental or more holistic nature.

Our results point to important implications for approaches to therapy. A change from lexical to segmental reading may be related to improved monitoring mechanisms. As shown in DH and ST, two cases with an initially large number of visually based lexical errors or mild speech apraxia, respectively, a segmental reading approach may support the buildup of monitoring mechanisms, leading to a more efficient word identification process.

In any case, an optimal therapeutic strategy should rely on the best possible diagnostic support for planning and evaluation. Based on our results, we suggest that in addition to standard linguistic error analysis, the online monitoring of word processing via eye tracking methodology should become a regular part of future diagnostic assessment batteries for patients with acquired dyslexia.

Original manuscript received 2 December 2013

Manuscript accepted 12 February 2014

First published online 18 March 2014

\section{REFERENCES}

Ablinger, I., Abel, S., \& Huber, W. (2008). Deep dysphasia as a phonetic input deficit: Evidence from a single case. Aphasiology, 22, 537-556. doi:10.1080/02687030701495403

Ablinger, I., Huber, W., Schattka, K., \& Radach, R. (2013). Recovery in a letter-by-letter reader: More efficiency at the expense of normal reading strategy. Neurocase, 19, 236-255. doi:10.1080/ 13554794.2012.667119

Ablinger, I., Weisse, K., Vorstius, C., Halm, K., Huber, W., \& Radach, R. (submitted). An eye movement based reading intervention in lexical and segmental readers with acquired dyslexia.

Baron, R., Hanley, J. R., Dell, G. S., \& Kay, J. (2008). Testing single- and dual-route computational models of auditory repetition with new data from six aphasic patients. Aphasiology, 22, 62-76. doi:10.1080/02687030600927092

Behrmann, M., Shomstein, S. S., Black, S. E., \& Barton, J. J. (2001). The eye movements of pure alexic patients during reading and nonreading tasks. Neuropsychologia, 39, 983-1002. doi:10.1016/S0028-3932 (01)00021-5 
Bowes, K., \& Martin, N. (2007). Longitudinal study of reading and writing rehabilitation using a bigraphbiphone correspondence approach. Aphasiology, 21, 687-701. doi:10.1080/02687030701192117

Buchanan, L., McEwen, S., Westbury, C., \& Libben, G. (2003). Semantics and semantic errors: Implicit access to semantic information from words and nonwords in deep dyslexia. Brain and Language, 84, 65-83. doi:10.1016/S0093-934X(02)00521-7

Cherney, L. R. (2004). Aphasia, alexia and oral reading. Topics in Stroke Rehabilitation, 11, $22-36$. doi:10.1310/VUPX-WDX7-J1EU-00TB

Coltheart, M. (1980). Deep dyslexia: A review of the syndrome. In M. Coltheart, K. Patterson, \& J. Marshall (Eds.), Deep dyslexia (pp. 22-48). London: Routledge \& Kegan Paul.

Coltheart, M. (2006). Acquired dyslexias and the computational modelling of reading. Cognitive Neuropsychology, 23, 96-109. doi:10.1080/02643290500202649

Coltheart, M. (2012). Dual-route theories of reading aloud. In J. Adelman (Eds.), Visual word recognition (Vol. 1, pp. 3-27). New York, NY: Psychology Press.

Coltheart, M., \& Byng, S. (1989). A treatment of surface dyslexia. In X. Seron \& G. Deloche (Eds.), Cognitive approaches in neuropsychological rehabilitation (pp. 159-174). Hillsdale, NJ: Lawrence Erlbaum Associates.

Coltheart, M., Rastle, K., Perry, C., Langdon, R., \& Ziegler, J. (2001). DRC: A dual route cascaded model of visual word recognition and reading aloud. Psychological Review, 108, 204-256. doi:10.1037/0033295X.108.1.204

Crisp, J., Howard, D., \& Lambon Ralph, M. A. (2011). More evidence for a continuum between phonological and deep dyslexia: Novel data from three measures of direct orthography-to-phonology translation. Aphasiology, 25, 615-641. doi:10.1080/02687038.2010.541470

Crisp, J., \& Lambon Ralph, M. A. (2006). Unlocking the nature of the phonological-deep dyslexia continuum: The keys to reading aloud Are in phonology and semantics. Journal of Cognitive Neuroscience, 18, 348-362. doi:10.1162/jocn.2006.18.3.348

De Partz, M. (1986). Re-education of a deep dyslexic patient: Rationale of the method and results. Cognitive Neuropsychology, 3, 149-177. doi:10.1080/02643298608252674

Dell, G. S., Martin, N., \& Schwartz, M. F. (2007). A case-series test of the interactive two-step model of lexical access: Predicting word repetition from picture naming. Journal of Memory and Language, 56, 490-520. doi:10.1016/j.jml.2006.05.007

Engbert, R., \& Krügel, A. (2010). Readers use Bayesian estimation for eye movement control. Psychological Science, 21, 366-371. doi:10.1177/0956797610362060

Friedman, R. B. (1996). Recovery from deep alexia to phonological alexia: Points on a continuum. Brain and Language, 52, 114-128. doi:10.1006/brln.1996.0006

Friedman, R. B., \& Nitzberg Lott, S. (2000). Rapid word identification in pure alexia is lexical but not semantic. Brain and Language, 72, 219-237. doi:10.1006/brln.2000.2286

Friedman, R. B., \& Nitzberg Lott, S. (2002). Successful blending in a phonological reading treatment for deep alexia. Aphasiology, 16, 355-372. doi:10.1080/02687040143000627

Friedman, R. B., \& Robinson, S. R. (1991). Whole-word training therapy in a stable surface alexic patient: It works. Aphasiology, 5, 521-527. doi:10.1080/02687039108248557

Greenwald, M. L. (2000). The acquired dyslexias. In S. E. Nadeau, B. A. Crosson, \& G. L. GonzalezRothi (Eds.), Aphasia and language: Theory to practice (pp. 159-183). New York, NY: The Guilford Press.

Hebbema, J. D. F., Hermans, J., \& van den Broek, K. (1974). A stepwise discriminant analysis program using density estimation. In G. Bruckmann (Ed.), Proceedings in computational statistics (pp. 101-110). Wien: Physica-Verlag.

Houghton, G., \& Zorzi, M. (2003). Normal and impaired spelling in a connectionist dual-route architecture. Cognitive Neuropsychology, 20, 115-162. doi:10.1080/02643290242000871

Huber, W., Poeck, K., Weniger, D., \& Willmes, K. (1983). Der Aachener Aphasie Test (AAT). Göttingen: Hogrefe.

Huber, W., Springer, L., \& Willmes, K. (1993). Approaches to aphasia therapy in Aachen. In A. Holland \& M. Forbes (Eds.), World perspectives on aphasia (pp. 55-86). San Diego, CA: Singular.

Huestegge, L., Radach, R., Corbic, D., \& Huestegge, S. M. (2009). Oculomotor and linguistic determinants of reading development: A longitudinal study. Vision Research, 49, 2948-2959. doi:10.1016/j. visres.2009.09.012

Hyönä, J., Bertram, R., \& Pollatsek, A. (2004). Are long compound words identified serially via their constituents? Evidence from an eye-movement-contingent display change study. Memory \& Cognition, 32, 523-532. doi:10.3758/BF03195844 
Inhoff, A. W., Eiter, B., \& Radach, R. (2005). Time course of linguistic information extraction from consecutive words during eye fixations in reading. Journal of Experimental Psychology: Human Perception and Performance, 31, 979-995. doi:10.1037/0096-1523.31.5.979

Inhoff, A. W., \& Radach, R. (1998). Definition and computation of oculomotor measures in the study of cognitive processes. In G. Underwood (Ed.), Eye guidance in reading and scene perception (pp. 29-53). Oxford: Elsevier.

Inhoff, A. W., Radach, R., \& Heller, D. (2000). Complex compounds in German: Interword spaces facilitate segmentation but hinder assignment of meaning. Journal of Memory and Language, 42, 23-50. doi:10.1006/jmla.1999.2666

Inhoff, A. W., Solomon, M., Radach, R., \& Seymour, B. (2011). Temporal dynamics of the eye voice span and eye movement control during oral reading. Journal of Cognitive Psychology, 23, 543-558. doi:10.1080/20445911.2011.546782

Johnson, R. L., \& Rayner, K. (2007). Top-down and bottom-up effects in pure alexia: Evidence from eye movements. Neuropsychologia, 45, 2246-2257. doi:10.1016/j.neuropsychologia.2007.02.026

Joseph, H. S., Liversedge, S. P., Blythe, H. I., White, S. J., \& Rayner, K. (2009). Word length and landing position effects during reading in children and adults. Vision Research, 49, 2078-2086. doi:10.1016/j. visres.2009.05.015

Kiran, S., Thompson, C. K., \& Hashimoto, N. (2001). Training grapheme to phoneme conversion in patients with oral reading and naming deficits: A model-based approach. Aphasiology, 15, 855-876. doi:10.1080/02687040143000258

Leff, A., \& Behrmann, M. (2008). Treatment of reading impairment after stroke. Current Opinion in Neurology, 21, 644-648. doi:10.1097/WCO.0b013e3283168dc7

Liversedge, S. P., \& Findlay, J. M. (2000). Saccadic eye movements and cognition. Trends Incognitive Sciences, 4, 6-14. doi:10.1016/S1364-6613(99)01418-7

Mitchum, C., \& Berndt, R. (1991). Diagnosis and treatment of the non-lexical route in acquired dyslexia: An illustration of the cognitive neuropsychological approach. Journal of Neurolinguistics, 6, 103-137. doi:10.1016/0911-6044(91)90003-2

Morton, J., \& Patterson, K. E. (1980). A new attempt at an interpretation, or, an attempt at a new interpretation. In M. Coltheart, K. E. Patterson, \& J. C. Marshall (Eds.), Deep dyslexia. London: Lawrence Erlbaum Associates.

Nickels, L. (1992). The autocue? Self-generated phonemic cues in the treatment of a disorder of reading and naming. Cognitive Neuropsychology, 9, 155-182. doi:10.1080/02643299208252057

Nolan, K. A., Volpe, B. T., \& Burton, L. A. (1997). The continuum of deep/surface dyslexia. Journal of Psycholinguistic Research, 26, 413-424. doi:10.1023/A:1025078003177

Oldfield, R. C. (1971). The assessment and analysis of handedness: The Edinburgh inventory. Neuropsychologia, 9, 97-113. doi:10.1016/0028-3932(71)90067-4

Patterson, K. E., Marshall, J. C., \& Coltheart, M. (1985). Surface dyslexia: Neuropsychological and cognitive studies of phonological reading. London: Lawrence Erlbaum Associates.

Radach, R., \& Heller, D. (2000). Relations between spatial and temporal aspects of eye movement control. In A. Kennedy, R. Radach, D. Heller, \& J. Pynte (Eds.), Reading as a perceptual process. Oxford: Elsevier.

Radach, R., Inhoff, A. W., \& Heller, D. (2004). Orthographic regularity gradually modulates saccade amplitudes in reading. European Journal of Cognitive Psychology, 16, 27-51. doi:10.1080/ 09541440340000222

Radach, R., \& Kempe, V. (1993). An individual analysis of initial fixation positions in reading. In G. d'Ydevalle \& J. van Rensbergen (Eds.), Perception and cognition. Advances in eye movement research. Amsterdam: Elsevier.

Radach, R., \& Kennedy, A. (2004). Theoretical perspectives on eye movements in reading: Past controversies, current issues, and an agenda for future research. European Journal of Cognitive Psychology, 16, 3-26. doi:10.1080/09541440340000295

Radach, R., \& Kennedy, A. (2013). Eye movements in reading: Some theoretical context. The Quarterly Journal of Experimental Psychology, 66, 429-452. doi:10.1080/17470218.2012.750676

Radach, R., \& McConkie, G. W. (1998). Determinants of fixation positions in words during reading. In G. Underwood (Ed.), Eye guidance in reading and scene perception. Oxford: Elsevier.

Rapcsak, S., Henry, M., Teague, S., Carnahan, S., \& Beeson, P. (2007). Do dual-route models accurately predict reading and spelling performance in individuals with acquired alexia and agraphia?. Neuropsychologia, 45, 2519-2524. doi:10.1016/j.neuropsychologia.2007.03.019

Rastle, K., Tyler, L. K., \& Marslen-Wilson, W. (2006). New evidence for morphological errors in deep dyslexia. Brain and Language, 97, 189-199. doi:10.1016/j.band1.2005.10.003 
Rayner, K. (1979). Eye guidance in reading: Fixation locations within words. Perception, 8, 21-30. doi: $10.1068 / \mathrm{p} 080021$

Rayner, K. (2009). Eye movements and attention in reading, scene perception, and visual search. The Quarterly Journal of Experimental Psychology, 62, 1457-1506. doi:10.1080/17470210902816461

Rayner, K., \& Johnson, R. L. (2005). Letter-by-letter acquired dyslexia is due to the serial encoding of letters. Psychological Science, 16, 530-534. doi:10.1111/j.0956-7976.2005.01570.x

Schattka, K. I., Radach, R., \& Huber, W. (2010). Eye movement correlates of acquired central dyslexia. Neuropsychologia, 48, 2959-2973. doi:10.1016/j.neuropsychologia.2010.06.005

Schotter, E. R., Angele, B., \& Rayner, K. (2012). Parafoveal processing in reading. Attention, Perception, \& Psychophysics, 74, 5-35. doi:10.3758/s13414-011-0219-2

Stadie, N., \& Rilling, E. (2006). Evaluation of lexically and nonlexically based reading treatment in a deep dyslexic. Cognitive Neuropsychology, 23, 643-672. doi:10.1080/02643290500538364

Tang, S., Reilly, R., \& Vorstius, C. (2012). EyeMap: A software system for visualizing and analyzing eye movement data in reading. Behavior Research Methods, 44, 420-438. doi:10.3758/s13428-011-0156-y

Vitu, F., McConkie, G. W., Kerr, P., \& O'Regan, J. K. (2001). Fixation location effects on fixation durations during reading: An inverted optimal viewing position effect. Vision Research, 41, 3513-3533. doi:10.1016/S0042-6989(01)00166-3

Vorstius, C., Radach, R., \& Lonigan, C. J. (in press). Eye movements in developing readers: A comparison of silent and oral sentence reading. Visual Cognition.

Weekes, B., \& Coltheart, M. (1996). Surface dyslexia and surface dysgraphia: Treatment studies and their theoretical implications. Cognitive Neuropsychology, 13, 277-315. doi:10.1080/026432996382033

Willmes, K. (1985). An approach to analyzing a single subject's scores obtained in a standardized test with application to the Aachen aphasia test (AAT). Journal of Clinical and Experimental Neuropsychology, 7, 331-352. doi:10.1080/01688638508401268

Yampolsky, S., \& Waters, G. (2002). Treatment of single word oral reading in an individual with deep dyslexia. Aphasiology, 16, 455-471. doi:10.1080/02687030244000068 